\title{
Comparative Analysis of Emotional Symptoms in Elderly Koreans with Hwa-Byung and Depression
}

\author{
Chae-Sung Im, Sengmi Baeg, Jin-Hoon Choi, Miji Lee, Hyun-Jin Kim, \\ Ik-Seung Chee, So-Hyun Ahn ${ }^{凶}$, and Jeong Lan Kim ${ }^{\bowtie}$ \\ Department of Psychiatry, Chungnam National University Hospital, Daejeon, Rupublic of Korea
}

\begin{abstract}
Objective This study compared the symptomatic emotional traits of elderly South Korean patients with hwa-byung and those with depression.

Methods We enrolled 58 patients with hwa-byung, 180 patients with depression, and 181 healthy control subjects. All participants completed the Hwa-byung Scale, Hamilton Depression Rating Scale (HDRS), Beck Depression Inventory (BDI), Geriatric Depression Scale (GDS), State Trait Anger Expression Inventory (STAXI), Reaction Inventory (RI), and Aggression Questionnaire (AQ). Chi-square tests and a one-way analysis of variance with Games-Howell post-hoc tests were used to compare demographic variables and scores.

Results A binary logistic regression analysis was used to examine risk factors for hwa-byung. Scores in the hwa-byung group were higher than those in the depression group for the HDRS; BDI; GDS; trait anger STAXI subscale (trait anger temperament and trait anger reaction); state anger and anger expression STAXI subscales (anger-in, anger-out, and anger control); physical and verbal aggression as well as anger and hostility AQ subscales; and disturbance, embarrassing circumstances, personal disrespect, and unpleasant encounters RI subscales. A binary logistic regression analysis demonstrated that the state anger STAXI subscale, verbal aggression and anger AQ subscales, and unpleasant encounters RI subscale were significantly associated with hwa-byung.

Conclusion Elderly patients with hwa-byung had more severe anger traits and states as well as higher depression severity compared to those diagnosed with clinical depression. Excessive anger and anger reactivity to unpleasant factors may be risk factors for hwa-byung, whereas the appropriate expression (rather than suppression) of anger may decrease the risk of hwa-byung.
\end{abstract}

Psychiatry Investig 2017;14(6):864-870

Key Words Anger, Aggression, Depression, Aged, Cultural psychiatry.

\section{INTRODUCTION}

Hwa-byung (anger syndrome) is a psychiatric condition wherein patients suppress rather than express negative emotions such as anger. Anger has unique features in Korean culture, ${ }^{1}$ such that hwa-byung is classified as a culture-related syndrome in the Diagnostic and Statistical Manual of Men-

Received: Apri 5, 2017 Accepted: July 2, 2017

Available online: October 13, 2017

$\triangle$ Correspondence: Jeong Lan Kim, MD, PhD

Department of Psychiatry, Chungnam National University Hospital, 282 Munhwa-ro, Jung-gu, Daejeon 35015, Republic of Korea

Tel: +82-42-280-7287, Fax: +82-42-280-7886, E-mail: kimjl@cnu.ac.kr

$\triangle$ Correspondence: So-Hyun Ahn, MD

Department of Psychiatry, Chungnam National University Hospital, 282 Munhwa-ro, Jung-gu, Daejeon 35015, Republic of Korea

Tel: +82-42-280-7281, Fax: +82-42-280-7886

E-mail: doctorahn99@hanmail.net

(c) This is an Open Access article distributed under the terms of the Creative Commons Attribution Non-Commercial License (http://creativecommons.org/licenses/bync/4.0) which permits unrestricted non-commercial use, distribution, and reproduction in any medium, provided the original work is properly cited. tal Disorders, Fourth Edition (DSM-IV). ${ }^{2}$ Hwa-byung usually develops in people who suppress their anger in repetitive conflict situations over a long period of time to avoid endangering amicable social relationships. ${ }^{3}$ Generally, individuals with hwa-byung recognize anger as the cause of their illness, ${ }^{4}$ but use defense mechanisms such as suppression, inhibition, and compliance to cope. ${ }^{3}$ While anger is the principal psychological symptom of hwa-byung, other symptoms include fatigue, panic, fear of impending death, depressive affectivity, indigestion, anorexia, shortness of breath, tachycardia, systemic pain, and the sensation of a lump in the epigastric region. ${ }^{2}$ Elderly women and individuals with lower educational and economic status are at higher risk for developing hwabyung compared to the general population. ${ }^{5}$

Anger is defined as an emotion that includes a state of physiological arousal and coexists with imaginative or intentional behaviors that culminate in inflicting injury on others. ${ }^{6} \mathrm{Al}-$ ternatively, aggression is defined as destructive and punitive 
behavior towards others. ${ }^{6}$ There is a significant correlation between anger and aggression; people who exhibit higher trait anger are more likely to react aggressively. ${ }^{6}$ If anger and aggression are not well-controlled in terms of intensity, frequency, and duration, they can produce negative effects on health, such as high blood pressure, cardiovascular diseases, ${ }^{7}$ pain, ${ }^{7,8}$ digestive symptoms, ${ }^{8}$ and mental disorders including hwa-byung, ${ }^{9}$ depression, ${ }^{10}$ dementia, ${ }^{11}$ and suicide. ${ }^{12}$

Until recently, hwa-byung was generally regarded as a common emotional expression or variant of depression among Koreans. ${ }^{9}$ Thus, most hwa-byung patients are diagnosed with depression in accordance with the DSM-IV criteria in clinical settings. ${ }^{13}$ A recent study examined differences between hwabyung and clinical depression and determined that hwa-byung was more likely to be a unique psychiatric syndrome rather than a variant of depression. ${ }^{1}$ However, few studies have examined the differences between anger and aggression in hwabyung and depression. Moreover, despite the fact that hwabyung is more prevalent in elderly people, no study to date has examined these differences in elderly patients. Therefore, we investigated differences in anger, aggression, and depression severity in elderly South Koreans with hwa-byung and depression.

\section{METHODS}

\section{Subjects}

Elderly (aged 60 or older) South Korean patients with hwabyung were recruited from an outpatient psychiatric clinic and the local community between June 2009 and February 2010. After a screening interview performed by a psychiatrist, 58 subjects ( 5 men and 53 women) met the hwa-byung scale and diagnostic criteria 13 for hwa-byung and were enrolled in the study. The depression group consisted of 180 elderly patients (44 men and 136 women) who had visited an outpatient psychiatric clinic between June 2009 and February 2010 and received a diagnosis of depressive disorder in accordance with the DSM-IV diagnostic criteria.

A psychiatrist administered a structured screening interview to all participants using the Mini International Neuropsychiatric Interview (MINI). ${ }^{14}$ Exclusion criteria were as follows: depressive episodes of bipolar disorder and psychotic symptoms; Parkinson's disease; cancer; stroke; neurologic disease including normal-pressure hydrocephalus; cognitive disorders including dementia accompanied by depression; a history of alcoholism or drug dependence; and refusal to participate in the study.

The healthy controls were 181 people aged 60 age or older who lived in the local community. To identify any psychiatric disorders in this group, a psychiatrist conducted a structural interview using the MINI. ${ }^{14}$ Those who had a mood disorder (including depression, psychosis, an anxiety disorder, a substance-related disorder, or dementia) or declined to participate in this study were excluded.

All subjects were provided with a written document sufficiently explaining the purpose and methods of the study and subsequently provided written informed consent. This study was approved by the Institutional Review Board of Chungnam National University Hospital (approval number 0907-64).

\section{Assessment tools}

\section{Hwa-Byung Scale ${ }^{13}$}

The Hwa-byung Scale was developed to diagnose hwa-byung in clinical and research settings. The scale consists of 3 groups, designated by letters A through C. Group A evaluates 6 core hwa-byung symptoms on a scale of 1-5 (subjective anger, feelings of unfairness, expressed anger, heat sensation, hostility, and "haan," a culturally Korean feeling of oppression by undefeatable forces). Group B evaluates 8 hwa-byung-related physical and behavioral symptoms on a scale of 1-3 (pushing up in the chest, epigastric mass, respiratory stuffiness, palpitation, dry mouth, sighing, many thoughts, and much pleading). Group C evaluates 8 other hwa-byung-related symptoms on a scale of $1-3$ or $1-5$ (sad mood/tearing, anxiety/agitation, feeling guilt, sleep disturbances, headache, anorexia, easily frightening, and going out). Higher scores signify a greater severity of hwa-byung symptoms.

\section{Korean Version of the Hamilton Depression Rating Scale}

The HDRS ${ }^{15}$ is the most widely used observer rating scale for depression. This scale consists of 17 questions. The KHDRS has been validated in previous studies. ${ }^{16}$ For each depressive symptom, 9 items are scored as $0-4$ points, and 8 items are scored as $0-2$. The range of total scores is $0-52$ points. A higher score means more severe depression.

\section{Korean Version of the Beck Depression Inventory}

The $\mathrm{BDI}^{17}$ is a self-reporting tool containing 21 questions about cognitive, emotional, motivational, and physical features of depression. For each depressive symptom, the range of responses is $0-3$ points, and a higher score means more severe depression. The K-BDI was previously validated by Han et al. ${ }^{18}$

\section{Korean Version of the Geriatric Depression Scale}

The GDS ${ }^{19}$ is a self-reporting tool used to evaluate depression in the elderly. This inventory consists of 30 yes-no questions. Unlike the BDI and HDRS, the GDS does not address 
physical symptoms of depression but rather focuses on cognitive symptoms. The K-GDS was previously validated by Cho et al. ${ }^{20}$

\section{State and Trait Anger Inventory ${ }^{21,22}$}

The STAXI is a self-rating tool used to measure the experience and expression of anger. This tool consists of 44 questions: 10 questions are about the state of anger, 10 questions are about traits of anger, and 24 questions regard anger expression. The trait anger section contains 5 questions about anger temperament and 5 questions about anger reactions. The anger expression section includes 8 questions about angerin, 8 questions about anger-out, and 8 questions about anger control. Higher scores for state anger and trait anger signify a greater intensity of anger, while higher scores for anger expression indicate a higher frequency of anger expression.

\section{Aggression Questionnaire ${ }^{23,24}$}

The AQ is a self-reporting scale used to assess aggression, anger, and hostility. The scale consists of 29 questions addressing 4 main factors: physical aggression, verbal aggression, anger, and hostility. ${ }^{24}$

\section{Reaction Inventory ${ }^{25}$}

The RI is a self-reporting scale used to evaluate circumstances that may cause anger. The scale consists of 76 questions addressing 4 factors: unpredictable disruptions and disturbances, embarrassing circumstances, personal disrespect, and unpleasant encounters. ${ }^{26}$

\section{Statistical analysis}

The chi-squared test and one-way analysis of variance (ANOVA) were used to compare demographic variables between the hwa-byung, depression, and control groups. A one-way ANOVA was also used to compare depression, anger, and aggression characteristics between the 3 groups. The Games-Howell test was used for post-hoc analyses. Finally, a logistic regression analysis was used to evaluate the effects of anger and aggression on the development of hwa-byung. All statistical analyses were performed using the Statistical Package for the Social Sciences (SPSS) version 12.0 for Windows (SPSS Inc., Chicago, IL, USA), and statistical significance was set at $\mathrm{p}<0.05$.

\section{RESULTS}

\section{Demographic characteristics of subjects}

The demographic characteristics of the hwa-byung group $(n=58)$, depression group $(n=180)$, and control group $(n=$ 181) are shown in Table 1. In all 3 groups, the percentage of women was significantly higher than that of men. The average age was significantly lower in the hwa-byung group than in the depression group or the control group. There were no differences in education, monthly income, marital status, or habitation status between the 3 groups.

\section{Comparison of depressive symptoms between the hwa-byung, depression, and control groups}

Between-group differences in the severity of depressive symptoms were examined using one-way ANOVA (Table 2). The hwa-byung group had significantly higher scores on the HDRS, BDI, and GDS than the depression and control groups. Furthermore, the depression group had significantly higher

Table 1. Participant demographic characteristics

\begin{tabular}{|c|c|c|c|c|}
\hline Characteristics & Hwa-byung $(\mathrm{N}=58)$ & Depression $(\mathrm{N}=180)$ & Control $(\mathrm{N}=181)$ & p-value \\
\hline $\operatorname{Sex}(\%)$ & & & & $<0.01^{*}$ \\
\hline Male & $5(8.6)$ & $44(24.4)$ & $67(37.0)$ & \\
\hline Female & $53(91.4)$ & $136(75.6)$ & $114(63.0)$ & \\
\hline Age, mean (SD) & $69.8(5.9)$ & $72.6(6.6)$ & $73.4(5.9)$ & $0.01^{\dagger}$ \\
\hline Education (years), mean (SD) & $4.2(4.3)$ & $4.1(4.0)$ & $4.4(4.4)$ & $0.85^{\dagger}$ \\
\hline Monthly income (10,000 Won), mean (SD) & $70.8(44.6)$ & $62.8(60.8)$ & $53.5(54.9)$ & $0.09^{\dagger}$ \\
\hline Marital status (\%) & & & & $0.58^{*}$ \\
\hline Married & $40(69.0)$ & $114(63.3)$ & $111(61.3)$ & \\
\hline Widowed, etc. $\ddagger$ & $18(31.0)$ & $66(36.7)$ & $70(38.7)$ & \\
\hline Habitation status (\%) & & & & $0.19^{*}$ \\
\hline Alone & $11(19.0)$ & $53(29.4)$ & $55(30.4)$ & \\
\hline Family without spouse & $9(15.5)$ & $13(7.2)$ & $13(7.2)$ & \\
\hline Spouse & $28(48.3)$ & $96(53.5)$ & $89(49.2)$ & \\
\hline Spouse and family & $10(17.2)$ & $18(10.0)$ & $24(13.3)$ & \\
\hline
\end{tabular}

${ }^{*}$ chi-square test, ${ }^{\dagger}$ one-way ANOVA, ${ }^{\ddagger}$ divorced/separation, unmarried. SD: standard deviation 
scores than the control group on the HDRS and GDS but not the BDI.

\section{Comparison of anger and aggression between the hwa-byung, depression, and control groups}

Total and subscale scores for the STAXI, AQ, and RI were compared between the 3 groups (Table 3). The hwa-byung group had significantly higher scores than the depression and control groups in STAXI total, trait anger, trait anger-temperament, state anger, AQ total, physical aggression, anger, hostility, and anger reaction to unpleasant encounters. The depression group had significantly lower scores than the hwabyung and control groups in STAXI total, trait anger, trait anger-reaction, anger expression, anger-in, anger-out, anger control, AQ total, verbal aggression, hostility, RI total, anger reaction to unpredictable disruptions and disturbances, anger reaction to embarrassing circumstances, and anger reaction to personal disrespect.

\section{Effects of anger and aggression on the development of hwa-byung}

To investigate the effects of anger and aggression on the development of hwa-byung, odds ratios (ORs) and 95\% confidence intervals (CIs) were determined using a logistic regression analysis of STAXI, AQ, and RI subscale scores (Table 4). The risk of hwa-byung increased 1.27-fold for every 1-point increase in state anger score on the STAXI. In contrast, the risk of hwa-byung increased 1.07-fold, 1.06-fold, 1.04-fold,

Table 2. Severity of depressive symptoms in the hwa-byung, depression, and control groups

\begin{tabular}{ccccccc}
\hline $\begin{array}{c}\text { Depressive symptom } \\
\text { scales }\end{array}$ & $\begin{array}{c}\text { Hwa-byung (N=58) } \\
{[\text { Mean (SD)] }}\end{array}$ & $\begin{array}{c}\text { Depression (N=180) } \\
{[\text { Mean (SD)] }}\end{array}$ & $\begin{array}{c}\text { Control (N=181) } \\
{[\text { Mean (SD)] }}\end{array}$ & \multicolumn{3}{c}{ Significance } \\
\cline { 5 - 7 } F-value & p-value & Post-hoc analysis \\
\hline HDRS & $17.0(6.5)$ & $10.7(5.7)$ & $4.8(4.8)$ & 120.1 & 0.02 & $1>2>3$ \\
BDI & $21.1(10.8)$ & $8.8(7.2)$ & $7.6(6.8)$ & 72.2 & $<0.01$ & $1>2,3$ \\
GDS & $15.1(5.9)$ & $12.2(4.3)$ & $10.7(2.8)$ & 26.2 & $<0.01$ & $1>2>3$ \\
\hline
\end{tabular}

Games-Howell tests were used for post-hoc analyses. 1: hwa-byung group, 2: depression group, 3: control group. SD: standard deviation, BDI: Beck Depression Inventory, GDS: Geriatric Depression Scale, HDRS: Hamilton Depression Rating Scale

Table 3. Comparison of anger and aggression scores in the hwa-byung, depression, and control groups

\begin{tabular}{|c|c|c|c|c|c|c|}
\hline \multirow{2}{*}{ Anger scales } & \multirow{2}{*}{$\begin{array}{c}\text { Hwa-byung }(\mathrm{N}=58) \\
{[\text { Mean }(\mathrm{SD})]}\end{array}$} & \multirow{2}{*}{$\begin{array}{c}\text { Depression }(\mathrm{N}=180) \\
{[\text { Mean }(\mathrm{SD})]}\end{array}$} & \multirow{2}{*}{$\begin{array}{c}\text { Controls }(\mathrm{N}=181) \\
{[\operatorname{Mean}(\mathrm{SD})]}\end{array}$} & \multicolumn{2}{|c|}{ Significance } & \multirow{2}{*}{ Post-hoc analysis } \\
\hline & & & & F-value & p-value & \\
\hline \multicolumn{7}{|l|}{ STAXI } \\
\hline Total & $76.7(14.2)$ & $61.5(9.0)$ & $71.2(1.2)$ & 60.3 & $<0.01$ & $1>3>2$ \\
\hline Trait anger & $20.3(0.3)$ & $15.4(5.1)$ & $17.9(6.1)$ & 20.4 & $<0.01$ & $1>3>2$ \\
\hline Temperament & $10.2(3.4)$ & $8.5(3.3)$ & $8.5(3.4)$ & 6.5 & 0.02 & $1>2,3$ \\
\hline Reaction & $10.1(0.1)$ & $6.9(2.9)$ & $9.4(3.6)$ & 35.9 & $<0.01$ & $1,3>2$ \\
\hline State anger & $12.5(4.7)$ & $10.2(1.6)$ & $10.2(0.7)$ & 29.8 & $<0.01$ & $1>2,3$ \\
\hline Anger expression & $44.1(8.8)$ & $35.9(5.2)$ & $32.0(6.7)$ & 65.5 & $<0.01$ & $1,3>2$ \\
\hline Anger-in & $13.1(4.4)$ & $9.8(2.6)$ & $11.1(3.6)$ & 30.4 & $<0.01$ & $1,3>2$ \\
\hline Anger-out & $13.4(5.1)$ & $10.6(4.0)$ & $12.2(4.2)$ & 12.0 & $<0.01$ & $1,3>2$ \\
\hline Anger control & $17.7(4.9)$ & $15.6(4.6)$ & $18.9(4.6)$ & 23.5 & $<0.01$ & $1,3>2$ \\
\hline \multicolumn{7}{|l|}{$\mathrm{AQ}$} \\
\hline Total & $64.2(16.9)$ & $50.1(11.9)$ & $55.1(13.5)$ & 25.1 & $<0.01$ & $1>3>2$ \\
\hline Physical aggression & $18.1(6.2)$ & $14.6(4.2)$ & $15.4(4.6)$ & 12.2 & $<0.01$ & $1>2,3$ \\
\hline Verbal aggression & $11.6(3.7)$ & $9.7(3.9)$ & $11.4(4.1)$ & 10.2 & $<0.01$ & $1,3>2$ \\
\hline Anger & $17.3(4.3)$ & $13.8(3.2)$ & $14.4(4.3)$ & 18.2 & $<0.01$ & $1>2,3$ \\
\hline Hostility & $17.3(5.9)$ & $12.1(4.3)$ & $14.0(4.3)$ & 28.7 & $<0.01$ & $1>3>2$ \\
\hline \multicolumn{7}{|l|}{ RI } \\
\hline Total & $212.1(57.6)$ & $146.9(40.6)$ & $199.0(60.4)$ & 58.7 & $<0.01$ & $1,3>2$ \\
\hline Disturbances & $27.6(9.4)$ & $17.8(6.0)$ & $25.9(8.8)$ & 60.8 & $<0.01$ & $1,3>2$ \\
\hline Embarrassing & $12.4(4.9)$ & $8.3(2.9)$ & $11.3(4.3)$ & 38.4 & $<0.01$ & $1,3>2$ \\
\hline Disrespect & $16.8(4.4)$ & $12.9(4.7)$ & $16.6(5.4)$ & 28.8 & $<0.01$ & $1,3>2$ \\
\hline Unpleasant & $5.7(2.1)$ & $4.1(1.9)$ & $4.5(2.1)$ & 12.8 & $<0.01$ & $1>2,3$ \\
\hline
\end{tabular}

1: hwa-byung group, 2: depression group, 3: control group. SD: standard deviation, AQ: Aggression Questionnaire, RI: Reaction Inventory, STAXI: State and Trait Anger Inventory 
Table 4. Logistic regression analysis assessing risk factors for hwa-byung

\begin{tabular}{lcccc}
\hline \multicolumn{1}{c}{ Anger scales } & Beta & SE & $\begin{array}{c}\text { Odds } \\
\text { ratio }\end{array}$ & $95 \%$ CI \\
\hline $\begin{array}{l}\text { STAXI } \\
\quad \text { Trait anger }\end{array}$ & & & & \\
$\quad$ Temperament & 0.068 & 0.059 & 1.07 & $0.954-1.202$ \\
$\quad$ Reaction & 0.061 & 0.061 & 1.06 & $0.942-1.198$ \\
$\quad$ State anger & 0.239 & 0.077 & $1.27^{*}$ & $1.092-1.477$ \\
Anger expression & & & & \\
$\quad$ Anger-in & 0.041 & 0.050 & 1.04 & $0.945-1.150$ \\
$\quad$ Anger-out & 0.003 & 0.051 & 1.00 & $0.908-1.108$ \\
$\quad$ Anger control & 0.009 & 0.041 & 1.01 & $0.931-1.095$ \\
AQ $\quad$ & & & & \\
Physical aggression & 0.003 & 0.043 & 1.00 & $0.923-1.091$ \\
$\quad$ Verbal aggression & -0.111 & 0.054 & $0.90^{*}$ & $0.805-0.994$ \\
Anger & 0.116 & 0.058 & $1.12^{*}$ & $1.001-1.259$ \\
Hostility & 0.070 & 0.042 & 1.07 & $0.988-1.163$ \\
RI $\quad$ & & & & \\
Disturbances & -0.019 & 0.037 & 0.98 & $0.913-1.054$ \\
Embarrassing & -0.035 & 0.056 & 0.99 & $0.896-1.115$ \\
Disrespect & -0.035 & 0.046 & 0.97 & $0.882-1.057$ \\
Unpleasant & 0.287 & 0.100 & $1.33^{*}$ & $1.096-1.622$ \\
\hline Chi-squared & & & & \\
\hline
\end{tabular}

Chi-squared $=76.2$, degrees of freedom $=14$. ${ }^{*} \mathrm{p}<0.05$. AQ: Aggression Questionnaire, CI: confidence interval, RI: Reaction Inventory, SE: standard error, STAXI: State and Trait Anger Inventory

and 1.01-fold for every 1-point increase in trait anger-temperament score, trait anger-reaction score, anger expression-angerin score, and anger control score, respectively. However, these increases were not statistically significant.

With regard to the $\mathrm{AQ}$, the risk of hwa-byung increased 1.12-fold for each 1-point increase in AQ total score, but decreased 0.90 -fold for each 1-point increase in verbal aggression score. These changes were statistically significant. The risk of hwa-byung increased 1.07-fold for each 1-point increase in hostility score, but this difference was not statistically significant.

For the RI, the risk of hwa-byung increased 1.33-fold for every 1-point increase in anger reaction to unpleasant encounters score, and this increase was statistically significant. In contrast, the risk of hwa-byung decreased 0.98 -fold, 0.99 fold, and 0.97 -fold for each 1-point increase in anger reaction to unpredictable disruptions and disturbances score, embarrassing circumstances score, and personal disrespect score, respectively. However, these results were not statistically significant.

\section{DISCUSSION}

In this study, we identified specific differences in the mag- nitude of anger, aggression, and depressive symptoms between elderly patients with hwa-byung, those with depression, and control subjects. Specifically, state anger score in the STAXI, verbal aggression and anger scores in the AQ, and unpleasant encounter scores in the RI were significantly associated with hwa-byung. Our data show clear differences in the clinical manifestations of hwa-byung and depression in elderly South Korean patients.

When comparing the severity of depressive symptoms between patients with hwa-byung, patients with depression, and control subjects, the hwa-byung group showed significantly higher scores on the observer-rated HDRS and the self-reported BDI and GDS scales than the depression and control groups. Notably, patients with hwa-byung or depression in this study displayed stabilized symptoms following pharmacological treatment as outpatients. It is therefore possible that patients with hwa-byung, who experience high degrees of anger and chiefly use defense mechanisms for emotional control, were more liberal in the evaluation of their depressive symptoms than were patients with depression. Min et al. ${ }^{27}$ suggested that comorbidity and mixed forms of anger, anxiety, and depressive disorders are quite common and that the chronic suppression of anger related to stress can lead to symptoms of depression. Min et al. ${ }^{27}$ specifically found that HDRS score was significantly correlated with most symptoms of hwa-byung. Although our study did not examine correlations between depressive scale items and hwa-byung symptoms, it is possible that the symptoms of hwa-byung influence depressive symptoms. As our study did not evaluate responsiveness to drug treatment, character, or defense mechanisms, these issues should be addressed in future studies comparing hwa-byung and depression.

We also compared the experience and expression of anger and aggression between different groups. Patients with hwabyung had significantly higher scores than depression patients for 3 STAXI subscales: state anger, which measures the baseline sensation of anger; trait anger, which assesses the tendency to feel anger and aggression during ordinary circumstances; and anger expression, which measures the expressive aspect of anger. Furthermore, patients with hwa-byung showed significantly higher scores than patients with depression for physical aggression, verbal aggression, anger, and hostility on the AQ. Patients with hwa-byung also showed significantly higher RI subscale scores than patients with depression. Notably, the RI measures anger reactions to circumstances that normally evoke anger, including unpredictable disruptions and disturbances, embarrassing circumstances, personal disrespect, and unpleasant encounters. These results are consistent with the results of other studies in adult patients with hwa-byung. ${ }^{3,28}$ Depression patients also had significantly 
lower scores than control subjects for anger reaction, anger-in, anger-out, and anger control on the STAXI; verbal aggression and hostility on the AQ; and anger reaction to unpredictable disruptions and disturbances, embarrassing circumstances, and personal disrespect on the RI. This is consistent with the idea that depression patients have an impaired ability to recognize internal emotional states including anger, ${ }^{29}$ and may be fearful of expressing anger. ${ }^{30}$ Future studies should examine this trait as a key factor for differentiating clinical depression from hwa-byung.

To investigate the effects of anger and aggression on the development of hwa-byung, ORs were computed using the STAXI, AQ, and RI subscales. For the STAXI, increases in state anger score significantly increased the risk of hwa-byung (OR: 1.27; 95\% CI: 1.092-1.477). On the AQ, increases in anger score also significantly increased the risk of hwa-byung (OR: 1.12; 95\% CI: 1.001-1.259), whereas increases in verbal aggression score decreased the risk of hwa-byung (OR: 0.90; 95\% CI: 0.805-0.994). For the RI, increases in the score for anger reaction to unpleasant encounters significantly increased the risk of hwa-byung (OR: 1.33; 95\% CI: 1.096-1.622). These relationships are in agreement with the concept that hwabyung is caused by a continued state of high anger, emotional suppression, and the partial or insufficient expression of anger and aggression. ${ }^{3}$ Therefore, hwa-byung may be preventable or treatable with education and re-training for the appropriate expression of feelings. These findings were obtained in a retrospective fashion, such that causal relationships with hwa-byung remain unclear. Future studies are needed to identify methods for the prevention and treatment of hwa-byung.

The present study had several limitations. First, small sample sizes limited the statistical power of our study. Second, there were significant differences in age and sex between the 3 groups, and subjects were sampled from different regions, such that our cohort may not be representative of the entire elderly population in South Korea. Third, we did not select patients based on whether they were receiving treatment, nor did we analyze medication data. Finally, although a hwabyung scale with verified utility and reliability was used to diagnose hwa-byung, the results in our cohort may not be accurate because the scale was not developed for patients over the age of 65 .

This study is the first of its kind to investigate differences in symptomatic manifestations of anger, aggression, and depression between elderly patients with hwa-byung, those with depression, and control subjects. Moreover, this study explored the effects of anger and aggression on the development of hwabyung. Elderly patients with hwa-byung generally showed significantly higher scores than depression patients on the HDRS, BDI, and GDS and on subscales of the STAXI, AQ, and
RI. Additionally, high scores on the state anger STAXI subscale, AQ anger subscale, and anger reaction to unpleasant encounters RI subscale were associated with an increased risk of hwa-byung, whereas a high score on the verbal aggression AQ subscale decreased the risk of hwa-byung. These findings identify emotional suppression as a pathological basis for hwa-byung and show important clinical differences between hwa-byung and depression that should be considered in future studies and clinical contexts.

\section{REFERENCES}

1. Min SK. Clinical correlates of hwa-byung and a proposal for a new anger disorder. Psychiatry Investig 2008;5:125-141.

2. American Psychiatric Association. Diagnostic Criteria from DSM-IVTR. Washington, D.C: American Psychiatric Association; 2000.

3. Min SK. A study on the concept of hwabyung. J Korean Neuropsychiatr Assoc 1989;28:604-615.

4. Chon KK, Park HK, Kim JW. Symptom interpretation in patients with hwabyung and hypertension. Korean J Health Psychol 1998;3:114-128.

5. Min SK, Lee MH, Kang HC, Lee HY. A clinical study of hwabyung. J Korean Med Assoc 1987;30:187-197.

6. Ramirez JM, Andreu JM. Aggression, and some related psychological constructs (anger, hostility, and impulsivity); some comments from a research project. Neurosci Biobehav Rev 2006;30:276-291.

7. Burns JW, Bruehl S, Caceres C. Anger management style, blood pressure reactivity, and acute pain sensitivity: evidence for "Trait x Situation" models. Ann Behav Med 2004;27:195-204.

8. Larsen RJ, Kasimatis M. Day-to-day physical symptoms: individual differences in the occurrence, duration, and emotional concomitants of minor daily illnesses. J Personal 1991;59:387-423.

9. Lin KM. Hwa-Byung: a Korean culture-bound syndrome? Am J Psychiatry 1983;140:105-107.

10. Gould RA, Ball S, Kaspi SP, Otto MW, Pollack MH, Shekhar A, et al. Prevalence and correlates of anger attacks: a two site study. J Affect Disord 1996;39:31-38.

11. Brodaty H, Low LF. Aggression in the elderly. J Clin Psychiatry 2003; 64(Suppl 4):36-43.

12. Jang JM, Park JI, Oh KY, Lee KH, Kim MS, Yoon MS, et al. Predictors of suicidal ideation in a community sample: roles of anger, self-esteem, and depression. Psychiatry Res 2014;216:74-81.

13. Min SK, Suh SY, Cho YK, Huh JE, Song KJ. Development of Hwabyung sclae and research criteria of hwabyung. J Korean Neuropsychiatr Assoc 2009;48:77-85.

14. Sheehan DV, Lecrubier Y, Sheehan KH, Amorim P, Janavs J, Weiller E, et al. The Mini-International Neuropsychiatric Interview (M.I.N.I.): the development and validation of a structured diagnostic psychiatric interview for DSM-IV and ICD-10. J Clin Psychiatry 1998;59(Suppl 20:22-33); quiz 34-57.

15. Hamilton M. A rating scale for depression. J Neurol Neurosurg Psychiatry 1960;23:56-62.

16. Yi JS, Bae SO, Ahn YM, Park DB, Noh KS, Shin HK, et al. Validity and reliability of the Korean version of the Hamilton Depression Rating Scale (K-HDRS). J Korean Neuropsychiatr Assoc 2005;44:456-465.

17. Beck AT, Ward CH, Mendelson M, Mock J, Erbaugh J. An inventory for measuring depression. Arch Gen Psychiatry 1961;4:561-571.

18. Han HM, Yeom TH, Shin YW, Kim KH, Yoon DJ, Jung KJ. A Stanardization study of beck depression inventory in Korea. J Korean Neuropsychiatr Assoc 1986;25:487-502.

19. Yesavage JA, Brink TL, Rose TL, Lum O, Huang V, Adey M, et al. Development and validation of a geriatric depression screening scale: a preliminary report. J Psychiatr Res 1982;17:37-49. 
20. Cho MJ, Bae JM, Suh GH, Hahm BJ, Kim JK, Lee DW, et al. Validation of Geriatric Depression Scale, Korean Version (GDS) in the assessment of DSM-III-R major depression. J Korean Neuropsychiatr Assoc 1999; 38:48-63.

21. Spielberger CD. State-Trait Anger Expression Inventory: Professional Manual. Odessa, Florida: Psychological Assessment Resources; 1988.

22. Chon KK, Kim DY, Yi JS. Development of the STAXI-K:IV. Korean J Art Ther 2000;7:33-50

23. Buss AH, Perry M. The aggression questionnaire. J Personal Soc Psychol 1992;63:452-459.

24. Seo SK, Kwon SM. Validation study of the Korean version of the aggression questionnaire. Korean J Clin Psychol 2002;21:487-501.

25. Evans DR, Jessup A, Hearn MT. Development of a reaction inventory to measure guilt. J Personal Assess 1975;39:421-423.
26. Cho YW, Kim JL, Kim SY, Wang SK, Chee IS. A standardization ofr the Korean version of the reaction inventory. J Korean Soc Biol Ther Psychiatry 2009;15:130-139.

27. Min SK, Suh SY, Song KJ. Symptoms to use for diagnostic criteria of hwa-byung, an anger syndrome. Psychiatry Investig 2009;6:7-12.

28. Chon KK, Kim JW, Park HK. Life stress and coping styles of hwabyung patients and healthy control. Korean J Stress Res 1998;6:9-23.

29. Gollan JK, Pane HT, Mc Closkey MS, Coccaro EF. Identifying differences in biased affective information processing in major depression. Psychiatry Res 2008;159:18-24.

30. Goldman L, Haaga DA. Depression and the experience and expression of anger in marital and other relationships. J Nerv Ment Dis 1995;183: 505-509. 